\title{
Assertional verification of a connection management protocol
}

\author{
András L. Oláh and Sonia M. Heemstra de Groot \\ TIOS Group, Dept. of Electrical Eng., University of Twente \\ POB 217, 7500 AE, Enschede, the Netherlands. \\ Telephone: +31-53-892819. Fax: +31-53-333815. \\ E-mail: olah@cs.utwente.nl
}

\begin{abstract}
This paper deals with the verification of a connection management protocol which uses timestamps and network-wide synchronized clocks for the reliable opening and closing of connections. We prove the correctness of the protocol over an unreliable, bounded-delay network for the cases when (i) timestamps are unbounded; (ii) timestamps are from a finite modulo- $N$. space. We determine the minimal safe value of $N$ as a function of realtime parameters such as maximum packet lifetime, clock skew and maximum connection duration.

The protocol is modeled as a state-transition system and we argue about the properties of the system using an assertional technique based on temporal logic. Apart from handling safety and progress properties, this framework is also suitable to describe real-time aspects of the system.
\end{abstract}

\section{Keywords}

transport protocol, connection management, timestamps; verification, temporal logic

\section{INTRODUCTION}

Transport protocols provide reliable communication between clients and servers over networks that can lose, reorder and duplicate messages. During the course of system execution, connections are opened and closed between the communicating peers. Connection management $(\mathrm{CM})$ is the function in transport protocols which handles the reliable opening and closing of connections.

One well known CM technique is the three-way handshake (Sunshine and Dalal, 1978). The disadvantage of the three-way handshake is that user data can only be delivered after an initial round-trip delay. The handshake can be eliminated if the server keeps 
track of the set of connection requests that are already accepted. If the identifier (ID) of a received request is not in the set of old IDs, the server accepts the request, and adds its ID to the set. The difference between $\mathrm{CM}$ protocols lies in the way of assigning the IDs to requests and the way of storing the set of old IDs. Several protocols have been proposed recently which are able to open connections without the three-way handshake such as CMSC (Biersack and Feldmeier, 1993), Delta- $t$ (Fletcher and Watson, 1978), and SCMP (Liskov et al, 1991).

SCMP (Synchronized Clock Message Protocol), which is the subject of the analysis in this paper, is based on the novel idea of using synchronized clocks (Liskov et al, 1991). The client associates a timestamp with each connection request and the server uses the associated timestamp to decide whether or not to accept a received request. If the server remembers the timestamp ts of the last accepted request from the client, then a request is new if its timestamp is greater than ts. However, it is not feasible to keep the last timestamp of every client due to the vast number of clients. Therefore, the server may discard state information of idle connections, but it maintains the variable upper which is the maximum of the discarded $t s$ values. If the server does not remember the latest timestamp from a client when a request arrives, then the request is accepted if this timestamp is greater than upper. Comparing the timestamp in the request to upper assures that duplicates are never accepted. On the other hand, a non-duplicate request may be rejected if its timestamp is less than upper. Due to the roughly synchronized clocks, the probability that a valid request has a timestamp lower than upper is low.

We do not model the clock synchronization protocol here. There are protocols that can synchronize clocks of computers even on a wide-area network to a few hundred milliseconds with very high probability, but there is no absolute guarantee that the clocks remain always synchronized. Therefore SCMP was designed in such a way that "if the rare event of unsynchronized clocks does occur, the protocol continues to work correctly, although there may be a degradation of performance" (Liskov et al, 1991).

The main result of our work is that we formally prove the above statement, namely that SCMP satisfies its safety requirements without assuming that the clocks are always synchronized. We consider the case of using unbounded timestamps and the case when timestamps are from a modulo- $N$ space. In the case of unbounded timestamps, the monotonicity of the clocks is the only requirement for the correct operation of SCMP.

When modulo- $N$ timestamps are used, we need to make further assumptions. Specifically, we have to assume that packet delays in the network, the duration of a connection incarnation, and the rate of the clocks are all bounded. These are not heavy assumptions because such assumptions have to be made for every reliable protocol that uses a bounded identifier space over a network which reorders packets (Shankar and Lee, 1993), (Watson, 1981). Notice that the bounded clock rate assumption does not imply synchronized clocks.*

From the practical viewpoint, the unbounded case models many situations very well. The number of microseconds in a year, for example, can be represented on 45 bits and one year is well beyond the expected packet lifetimes and connection durations in a real network. In cases when it is essential to keep the protocol headers as small as possible, our results for the modulo- $N$ case can be used to determine the smallest safe value of

\footnotetext{
*The difference between any two clocks should also be bounded for the clocks to be synchronized.
} 
$N$ from parameters of the network. From the theoretical viewpoint, we believe that our analysis provides further insight into the working of reliable protocols.

\subsection{Related work}

The designers of SCMP gave only informal arguments about the correctness of the protocol (Liskov et al, 1991). A formal treatment of SCMP is presented in (Lampson et $a l, 1993)$ where the correctness of SCMP, both safety and progress properties, is proven assuming unbounded timestamps and properly synchronized clocks. Neither the case of unsynchronized clocks nor the effects of using modulo- $N$ timestamps are considered. We take these into account in this paper, but we do not discuss liveness properties. CM protocols somewhat similar to SCMP are analyzed in (Shankar and Lee, 1993) taking also into account the finiteness of identifiers.

\subsection{Organization of paper}

We start in Section 2 by giving an introduction to our model. A state transition system is used to specify the behavior of the protocol and temporal logic to argue about its properties. Then in Section 3, we specify the version of SCMP that uses unbounded timestamps. We also introduce our safety requirements and prove that unbounded SCMP satisfies them even if the clocks are unsynchronized. The modulo- $N$ case is discussed in Section 4 where we derive sufficient constraints for the safety of modulo- $N$ SCMP. Some results from the case of unbounded SCMP are used during the verification of the modulo- $N$ version. Section 5 gives a short overview of how to model host failures. Section 6 concludes our paper. Many formal details are left out from this paper due to space limitations. For such details, we refer the reader to the full report (Oláh and Heemstra de Groot, 1995).

\section{FORMAL FRAMEWORK}

\subsection{State transition system}

The protocol and its environment can be described as a distributed system (Shankar, 1993). The processes in the system are the protocol entities and the communication channels. Each process is specified by a set of state variables, the initial state and a set of atomic events. Events are guarded commands in the form 'ec: condition; ac: action'. In any state, one of the events with true enabling condition is non-deterministically selected and its action is executed which results in a new state. The selection of the next event to be executed is influenced by the fairness requirements of the system (see e.g., Shankar, 1993).

State variables can be non-auxiliary or auxiliary. Non-auxiliary variables are used to describe the behavior of the system. Auxiliary variables, on the other hand, are only used to support the verification; they do not influence the behavior of the system in any way. The real-time aspects of the system are described by a special class of auxiliary variables, called epoch variables (Shankar, 1994). $\tau$ is a real-valued auxiliary variable which always 
gives the current (real) time. The epoch variables are used to store the value of $\tau$ at the occurrence of an event.

The state of channel $i$, denoted by $\mathbf{z}_{i}$, is specified by the sequence of packets currently in transit. The channels model an unreliable network, thus channels have events to drop, reorder and duplicate packets and these events are always enabled.

The protocol entities are modeled as processes which do not share state variables. Processes can communicate only through the channels. Each process can send packets to the channels originating from it and can receive packets from the channels ending in it.

\subsection{Temporal logic}

Each execution of a system is a behavior $\sigma=\left(s_{0}, e_{0}, s_{1}, e_{1}, s_{2}, \ldots\right)$, where $s_{i}$ denotes a state, $s_{0}$ is an initial state and every $\left(s_{i}, s_{i+1}\right)$ pair is a transition generated by the execution of the event $e_{i}$. The behavior $\sigma$ is an allowed behavior if it satisfies the fairness requirements. A system is characterized by the set of its allowed behaviors.

Temporal logic can be used to argue about behaviors (Lamport, 1994). Formulas in temporal logic are built up from state predicates and the temporal operator $\square$. The meaning of a formula is a Boolean-valued function on behaviors, defined as follows:

$$
\begin{aligned}
& P\left(s_{0}, e_{0}, s_{1}, \ldots\right)=P\left(s_{0}\right), \text { for any predicate } P . \\
& \square F\left(s_{0}, e_{0}, s_{1}, \ldots\right)=\left(\forall n: n \geq 0: F\left(s_{n}, e_{n}, s_{n+1}, \ldots\right)\right) \\
& \diamond F=\neg \square \neg F \\
& F \sim G=\square(F \Rightarrow \diamond G) \\
& F \rightarrow G=\square(F \Rightarrow \square G)
\end{aligned}
$$

Intuitively, a formula is an assertion about the program's behavior from some fixed time onwards. The formula $\square F$ (always $F$ ) asserts that $F$ is always true-that is, true now and at all future times. The formula $\diamond F$ (eventually $F$ ) asserts that $F$ is eventually true-that is, true now or at some future time. The formula $F \leadsto G(F$ leads to $G)$ asserts that if $F$ ever becomes true, then $G$ will be true then or at some later time. The formula $F \rightarrow G$ ( $F$ establishes $G$ ) asserts that if $F$ ever becomes true, then $G$ will be true then and at any later time.

The desired safety and liveness properties of a system as well as assumptions about the system are given as temporal formulas. A system is said to satisfy the assertion $F$ if $F$ evaluates to true for every allowed behavior. Well known proof rules (Lamport, 1994), (Shankar, 1993) can be used to show that a system satisfies a formula. 


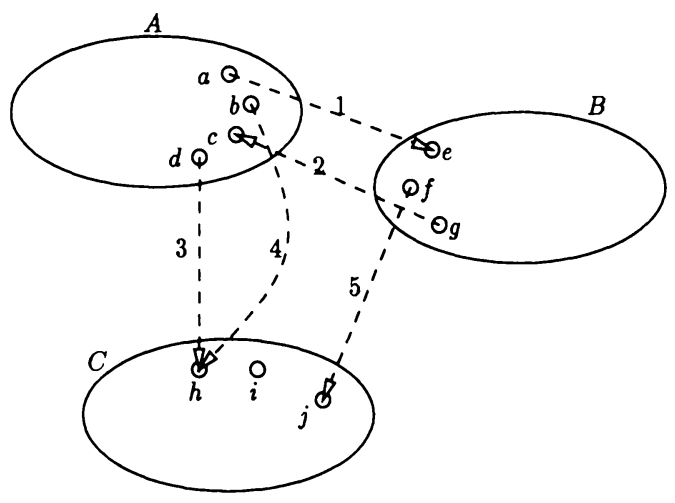

Figure 1 Endpoints, hosts and connections.

\section{SCMP WITH UNBOUNDED TIMESTAMPS}

\subsection{Protocol specification}

In our model, the communication takes place between connection endpoints. Each endpoint belongs to exactly one host, but a host may have several endpoints (Figure 1). Hosts model the physical entities (e.g. computers) on which the protocol executes.

Between each pair of endpoints there is a (potential) connection. The connections are asymmetric, one endpoint of a connection is called the client, the other is the server. Each connection may become open and closed many times over time. To distinguish among the different open periods of a connection we refer to connection incarnations. Every time a new attempt is made to open the connection, a new incarnation is started.

Figure 1 shows 3 hosts $(A, B, C)$; each of them has a number of connection endpoints. Host $A$, for example, has the endpoints $a, b, c$, and $d$. Connections take place between endpoints. There may be several connections between two hosts if they belong to different endpoints, e.g., connections 1 and 2 in the figure.

We use a Pascal-like notation to define the state variables, their initial values and the events. Hosts are denoted with capital letters in general. If we talk about a specific connection, then $C$ and $S$ are used to denote the host of the client and server endpoints of the connection in question, respectively. The notation H.time is used to refer the variable time of the host $H$. The same notation is used for accessing the header fields of a packet. The specification of a client and a server can be found in Table 1 and 2, respectively.

Every host, either client or server, maintains a monotonic clock time. Also, every host $H$ has a connection record $C R(a, b)$ for every $(a, b)$ pair, where $a$ and $b$ are different endpoint addresses and the host of $a$ is $H$. A CR contains the per-connection state information plus the auxiliary variables needed for the verification.

In the CR maintained by a client, status and $t s$ are non-auxiliary variables; lin, ts_sent and open_to are auxiliaries. status is one of closed, opening, open. ts contains the timestamp assigned to the current connection request when status $\neq$ closed. lin is the local incarnation counter, which is incremented every time a new connection opening is attempted. ts_sent 
State variables and their initial values:

time : integer $:=0$;

$C R(a, b):$ record of

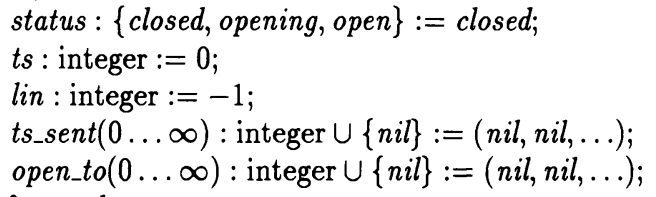

end of record;

Events:

ClkTick

ec: true

ac: for each $(a, b)$ pair do

if $C R(a, b)$. status $\neq$ closed $\wedge C R(a, b) . t s=$ time $-W_{C}$ then $C R(a, b)$. status $:=$ closed;

time ++ ;

Open $(a, b)$

ec: $\quad C R(a, b)$. status $=$ closed

ac: $\quad C R(a, b)$. status $:=$ opening;

$C R(a, b) . t s:=$ time;

$C R(a, b) \cdot \operatorname{lin}++$;

$C R(a, b) . t s \_s e n t(C R(a, b)$. lin $):=$ time;

$\operatorname{SendPkt}(a, b)$

ec: $\quad C R(a, b)$. status $=$ opening

ac: $\quad \operatorname{Send}(R: s r c=a, d s t=b, t s=C R(a, b) \cdot t s, \operatorname{lin}=C R(a, b) \cdot \operatorname{lin})$;

$\operatorname{RecvPkt}(A)$

ec: true

ac: $\quad$ if $C R(A . d s t, A . s r c) . s t a t u s=o p e n i n g \wedge C R(A . d s t, A . s r c) . t s=A . t s$ then

$C R(A . d s t, A . s r c)$. status $:=$ open;

$C R(A . d s t, A . s r c) . o p e n \_t o(C R(A . d s t, A . s r c) . l i n):=A . l i n ;$

Close $(a, b)$

ec: $\quad C R(a, b)$. status $\neq$ closed $\wedge C R(a, b)$. ts $<$ time

ac: $\quad C R(a, b)$. status $:=$ closed;

Table 1 The specification of the client 
and open_to are history variables which are used for expressing the desired properties of the protocol. ts_sent $(i)$ records the timestamp assigned to incarnation $i$ or nil if that incarnation does not exist yet. open_to $(i)$ records the remote incarnation to which the local incarnation $i$ was successfully opened or nil if that incarnation has not been open yet.

A server CR has the following variables: the state of the connection status which is either closed or open; and $t s$ which holds the timestamp of the latest accepted request. The rest of the variables are auxiliaries with almost identical meaning to the auxiliaries of the client CR. lin is the local incarnation number, ts_rcvd(i) stores the timestamp of the request accepted by incarnation $i$, and open_to $(i)$ is the remote incarnation to which the local incarnation was opened.

Apart from the per-connection CRs, each server maintains a non-auxiliary variable upper which gives an upper limit of the timestamps in the CRs of the closed connections.

The time variable is regularly incremented by the ClkTick event at both clients and servers. This event also enforces a maximum connection lifetime; when a connection becomes too old, then the connection is forcibly closed. $W_{C}$ and $W_{S}$ are the constants that give the maximum lifetime of a connection as measured on the clock of the client and server, respectively. The server also updates upper in the ClkTick event. The triggering of the ClkTick event is controlled by the clock synchronization protocol, but because this protocol is not modeled here, we simply enable the ClkTick events continuously. When necessary (see Section 4), we will use assumptions to bind the advance of the clocks to real time.

The C.Open $(a, b)$ event initiates a new connection incarnation. The status of the connection is changed to opening and the timestamp of the request is stored in the CR. While in the opening state, the client can (re)send the connection request. The fields of a connection request are the source and destination addresses src and $d s t$, the timestamp $t s$, and the client incarnation number lin.

When the server receives a request $R$, it looks up the appropriate $\mathrm{CR}$ using the source and destination of the request. If there is status information about this connection $(\text { status } \neq \text { closed })^{\dagger}$, then the request is a new one if R.ts > S.CR(R.dst, R.src).ts. Otherwise, the timestamp of the request is compared to upper. Messages that are 'newer' than time $+\epsilon$ are not accepted because they are too early. $\epsilon$ is a constant, which gives the maximum difference of the clocks when they are properly synchronized. If the request is acceptable, the server starts a new connection incarnation and enters the open state.

While in the open state, the server sends acknowledgments to the client. An acknowledgment contains a source, a destination, a timestamp and two auxiliary fields, the local and remote incarnation numbers. The client checks an incoming acknowledgment by comparing its timestamp to the timestamp stored in the CR. If the state of the connection is opening and the timestamps are equal, then the acknowledgment is accepted and the state is changed to open.

At the client, the state of a connection can only be changed to closed when the clock has advanced at least one tick since the connection was started. This is to prevent reusing the same timestamp in different connection incarnations.

A scenario when server $b$ receives a duplicate request from client $a$ is shown in Figure 2.

In an implementation the lack of a CR for the connection would mean the closed status, but in this model we cannot discard CRs because they also contain history information. 
State variables and their initial values:

time : integer $:=0$;

upper $:$ integer $:=0$;

$C R(a, b):$ record of

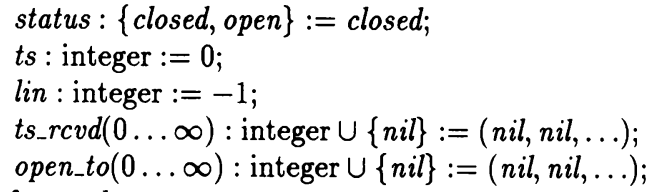

end of record;

Events:

ClkTick

ec: true

ac: for each $(a, b)$ pair do

if $C R(a, b)$. status $\neq$ closed $\wedge C R(a, b) . t s=$ time $-W_{S}$ then $C R(a, b)$. status $:=$ closed;

upper $:=\max \left(\right.$ upper, time $\left.-W_{S}\right)$;

time ++ ;

$\operatorname{RecvPkt}(R)$

ec: true

ac: if $C R(R . d s t, R . s r c) . s t a t u s=$ closed then

$l:=$ upper

else if $C R(R . d s t, R . s r c)$.status $\neq$ closed then

$l:=C R(R . d s t, R . s r c) . t s$

if $l<R$.ts $\leq$ time $+\epsilon$ then

$C R(R . d s t, R . s r c) . s t a t u s:=$ open;

$C R(R . d s t, R . s r c) . t s:=R . t s$

CR(R.dst, R.src).lin ++ ;

$C R(R . d s t, R . s r c) . t s \_r c v d(C R(R . d s t, R . s r c) . l i n):=R . t s$;

$C R(R . d s t, R . s r c) . o p e n \_t o(C R(R . d s t, R . s r c)$. lin $):=R . l i n ;$

SendPkt $(a, b)$

ec: $\quad C R(a, b)$. status $=$ open

ac: $\quad \operatorname{Send}(A: s r c=a, d s t=b, t s=C R(a, b) . t s$,

lin $=C R(a, b) \cdot$ lin, rin $=C R(a, b)$. open_to $(C R(a, b) \cdot \operatorname{lin}))$;

Close $(a, b)$

ec: $\quad C R(a, b)$. status $=$ open

ac: $\quad$ upper $:=\max ($ upper, $C R(a, b) . t s)$;

$C R(a, b)$. status $:=$ closed;

Table 2 The specification of the server 


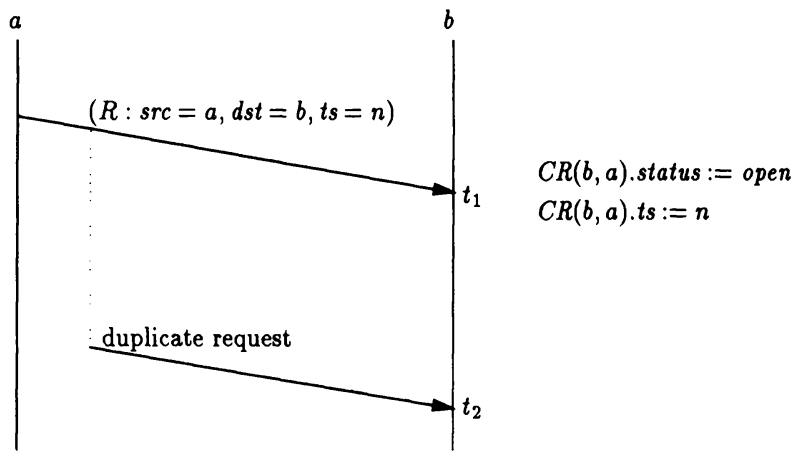

Figure 2 Duplicate request at the server.

The first copy of the packet is received at time $t_{1}$. The request is accepted, therefore status becomes open and the timestamp $n$ of the request is saved in $t s$. The duplicate is received at $t_{2}$. There are two cases to consider depending on the state of the server at $t_{2}$ :

- If the state is still open then the timestamp $n$ in the packet is compared to $C R(b, a)$.ts which is also equal to $n$, thus the request is rejected.

- If the state is closed, then there must have been a Close $(a, b)$ event between $t_{1}$ and $t_{2}$. This event assures that upper is at least $n$ after its occurrence. Therefore the duplicate request is rejected because its timestamp $n$ is less or equal to upper.

The duplicate is detected by the server because it has either the timestamp (when status = open) or an upper limit of the timestamp (when status $=$ closed) of the most recent request from the client.

\subsection{Desired safety properties}

The service provided by this specification is rather limited, therefore it should not be treated as a complete protocol. Our main goal was to model the mechanism by which SCMP can set up connections. In order to keep the discussion focused on this goal, several details have been omitted which can be easily added to this specification later on. The formal treatment of how an abstract $\mathrm{CM}$ protocol like this and a data-transfer protocol can be transformed into a full-featured transport protocol is given by (Shankar, 1991).

The definition of the desired safety properties which SCMP is expected to satisfy are expressed by the assertions $\mathcal{A}_{1-3}$, where the assertions should hold for all different $a, b$ endpoint addresses and for all $i, j, k$ integers.

$$
\begin{aligned}
& \mathcal{A}_{1} \equiv S . C R(a, b) . o p e n_{-} t o(i)=j \rightarrow C . C R(b, a) . o p e n_{-} t o(j) \in\{i, n i l\} \\
& \mathcal{A}_{2} \equiv C . C R(a, b) . o p e n_{-} t o(i)=j \rightarrow S . C R(b, a) . o p e n_{-} t o(j)=i \\
& \mathcal{A}_{3} \equiv S . C R(a, b) . o p e n_{-} t o(i)=j \neq n i l \wedge k \neq i \rightarrow S . C R(a, b) . o p e n_{-} t o(k) \neq j
\end{aligned}
$$

$\mathcal{A}_{1}$ requires that once the server incarnation $i$ becomes open to the client incarnation $j$, then at that and any later time $j$ is either open to $i$ or $j$ has not been open to any 
other incarnation. $\mathcal{A}_{2}$ is a similar statement, but from the client's viewpoint. If the client incarnation $i$ is open to the server incarnation $j$, then at that and any later time $j$ must be open to $i . \mathcal{A}_{3}$ requires that if the server incarnation $i$ is open to the client incarnation $j$, then there may be no other server incarnation $k$ which is also open to $j$.

To show that $\mathcal{A}_{1-3}$ are indeed sufficient requirements for a $\mathrm{CM}$ protocol, we have proven (Oláh and Heemstra de Groot, 1995) that $\mathcal{A}_{1-3}$ imply the generic invariant requirements for any CM service as given in (Shankar, 1991).

\subsection{Proving the safety of unbounded SCMP}

To prove that the invariance property $\square F$ is satisfied by a system, it is enough to show that

- $F$ is true in any of the initial states;

- $F$ is preserved by every event $e$ of the system, i.e. ( $\forall e:\{F\} e\{F\})$.

The difficulty is proving $\{F\} e\{F\}$ for every event. A usual approach is to formulate further assertions $F_{0}, F_{1}, \ldots F_{i}$, such that the conjunction of them satisfies the invariance rule. This requires the thorough understanding of how the system works. In our experience, the proper selection of the auxiliary variables is also an important factor in proving the invariance property. A heuristic approach to generate the assertions based on weakest preconditions is discussed by (Shankar, 1993).

In this subsection, we give the outline of the proof that unbounded SCMP satisfies the assertions $\mathcal{A}_{1-3}$ by presenting the assertions we used to prove the invariance of $\mathcal{A}_{1-3}$. Due to space limitations, we cannot go into details here but it should not cause any difficulty to fill out the missing details.

The assertions $\mathcal{A}_{4-6}$ provide the link between timestamps and incarnation numbers. $\mathcal{A}_{4}$ states that if there is a request $R$ in the network, then its timestamp $R$.ts is equal to the timestamp of the client incarnation $R$.lin which is stored in the history variable ts_sent of the corresponding CR. $\mathcal{A}_{5}$ says that if server incarnation $i$ is connected to the client incarnation $j$, then the timestamp $t_{-} r c v d(i)$ received by incarnation $i$ is equal to the timestamp ts_sent $(j)$ sent by incarnation $j . \mathcal{A}_{6}$ is similar to $\mathcal{A}_{4}$, but it is for acknowledgments: if there is an ack in the network then its rin and $t s$ fields are equal to the appropriate history variables of the server.

$$
\begin{aligned}
& \mathcal{A}_{4} \equiv R \in z \rightarrow R . t s=C . C R(R . s r c, R . d s t) . t s \_s e n t(R . l i n) \neq n i l \\
& \mathcal{A}_{5} \equiv S . C R(a, b) . o p e n \_t o(i)=j \rightarrow S . C R(a, b) . t s \_r c v d(i)=C . C R(b, a) . t s \_s e n t(j) \neq n i l \\
& \mathcal{A}_{6} \equiv A \in z \rightarrow\left(\text { A.rin }=S . C R(A . s r c, A . d s t) . o p e n_{-} t o(A . l i n) \neq \text { nil } \wedge\right. \\
& \left.A . t s=S . C R(A . s r c, A . d s t) . t s \_r c v d(A . l i n) \neq n i l\right)
\end{aligned}
$$

$\mathcal{A}_{5}$ and $\mathcal{A}_{6}$ are preconditions of the required properties $\mathcal{A}_{1}$ and $\mathcal{A}_{2}$ with respect to the C.RecvPkt event of the client. $\mathcal{A}_{5}$ is also a precondition of $\mathcal{A}_{6}$ with respect to the event S.SendPkt; $\mathcal{A}_{4}$ is the precondition of $\mathcal{A}_{5}$ with respect to S.RecvPkt. 
The following two assertions $\mathcal{A}_{7,8}$ state that the timestamps of successive incarnations are monotone increasing. $\mathcal{A}_{7}$ is a precondition of $\mathcal{A}_{2}$ with respect to C.RecvPkt. $\mathcal{A}_{8}$ is a precondition of $\mathcal{A}_{3}$ with respect to $S$.RecvPkt.

$$
\begin{aligned}
& \mathcal{A}_{7} \equiv \square\left(0 \leq i<C . C R(a, b) . \text { lin } \Rightarrow C . C R(a, b) . t s \_s e n t(i)<C . C R(a, b) . t s \_s e n t(i+1)\right) \\
& \mathcal{A}_{8} \equiv \square\left(0 \leq i<S . C R(a, b) . \text { lin } \Rightarrow S . C R(a, b) . t s_{-} r c v d(i)<S . C R(a, b) . t s_{-} r c v d(i+1)\right)
\end{aligned}
$$

Finally, $\mathcal{A}_{9-11}$ are assertions that are sufficient preconditions for the preservation of $\mathcal{A}_{7,8}$. $\mathcal{A}_{9}$ states that if a connection is closed as observed by the client, then the clock of the client is greater than the timestamp of the most recent incarnation. This is a precondition of $\mathcal{A}_{7}$ with respect to C.Open. $\mathcal{A}_{10,11}$ are the preconditions of $\mathcal{A}_{8}$ with respect to S.RecvPkt.

$$
\begin{aligned}
\mathcal{A}_{9} \equiv \square\left(C . C R(a, b) . s t a t u s=\text { closed } \Rightarrow C . C R(a, b) . t s \_s e n t(C . C R(a, b) . \text { lin })<C . t i m e\right) \\
\mathcal{A}_{10} \equiv \square(S . C R(a, b) . \text { status } \neq \text { closed } \Rightarrow \\
\left.\quad \text { S.CR }(a, b) . t s \_r c v d(S . C R(a, b) . \text { lin })=S . C R(a, b) . t s\right) \\
\mathcal{A}_{11} \equiv \square\left(S . C R(a, b) . \text { status }=\text { closed } \Rightarrow S . C R(a, b) . t s \_r c v d(S . C R(a, b) . \text { lin }) \leq \text { S.upper }\right)
\end{aligned}
$$

Extending $\mathcal{A}_{1-11}$ with some trivial assertions, one can prove that the safety requirements $\mathcal{A}_{1-3}$ are indeed invariants of unbounded SCMP. Unbounded SCMP satisfies these safety requirements without assuming that the clocks are synchronized.

\section{SCMP WITH MODULO- $N$ TIMESTAMPS}

In practice, protocols use a modulo- $N$ representation of identifiers. The use of modulo- $N$ timestamps in SCMP means that the same modulo- $N$ timestamp is assigned to different incarnations over time. It is clear that a timestamp can only be reused when the packets carrying the same timestamp from earlier incarnations have disappeared from the network. To allow the reuse of the timestamps, we will assume that the network does not delay packets longer than $M P L$ seconds.

Furthermore, we also have to assume that neither the client, nor the server keep a timestamp in use indefinitely. The server must not keep a connection open too long, otherwise a new incarnation started by the client using the same timestamp could misinterpret an old acknowledgment as an acknowledgment of the new incarnation. The client must also limit the lifetime of an incarnation in order to allow the server to discard the CR of old connections. The constants $W_{C}$ and $W_{S}$ in the specification give the maximum lifetime of a client and server incarnation, respectively.

$W_{C}$ and $W_{S}$ are given as the number of ticks of the local clock. In order to be useful for limiting the lifetime of an incarnation, the rates of the clocks have to be bound to the real time. Therefore, we will assume that the rate of any clock is within certain bounds. Our goal is to show that the limited packet lifetime and bounded clock skew are sufficient conditions for the safety of modulo- $N$ SCMP.

The following technique is proposed in (Shankar and Lee, 1993) to formally verify a modulo- $N$ protocol: 
- Define sufficient conditions under which the unbounded identifiers can be replaced by modulo- $N$ identifiers without affecting the protocol's behavior. The conditions are called correct interpretation (CI) conditions.

- Prove that the unbounded protocol satisfies the CI conditions.

The test $a>b$ of unbounded numbers can be replaced by an equivalent test on their modulo- $N$ counterparts if

$$
(\exists K: K<N: \square(b+K \geq a \geq b+K-N+1))
$$

holds. The equivalent modulo- $N$ test is $K \geq a \ominus b \geq 1$, where $\ominus$ means modulo- $N$ subtraction.

To apply this technique for SCMP, we formulated the CI conditions and derived necessary requirements for their invariance. It turned out that the clocks must be synchronized if we want the protocol to satisfy the CI conditions. To see why, let us consider the $S . R e c v P k t(R)$ event. The server uses its local clock S.time to check if $R$ is a recent request, hence there is a test $R . t s \leq S$.time $+\epsilon$. Applying (1) for this case, we get

$$
(\exists K: K<N: \square(R \in z \Rightarrow S . t i m e+\epsilon+K \geq R . t s \geq S . t i m e+\epsilon+K-N+1))
$$

Since a request $R$ gets its timestamp from the clock $C$.time of the client, the above formula can only be satisfied by the protocol if the difference of the clocks is bounded. However, this is not what we wanted to achieve because our goal was to prove that modulo- $N$ SCMP is safe even if the clocks are not synchronized.

Let us notice that the CI conditions are sufficient conditions for the equivalence of bounded and modulo- $N$ SCMP, i.e., for every behavior $\sigma$ of unbounded SCMP there exists a behavior $\sigma^{\prime}$ of modulo- $N$ SCMP which consists of the same sequence of states (modulo- $N$, of course) and vice versa. For the safety of modulo- $N$ SCMP, however, it is sufficient if every behavior $\sigma^{\prime}$ of modulo- $N$ SCMP satisfies the safety requirements $\mathcal{A}_{1-3}$.

Realizing this, we can try to replace the CI conditions with weaker conditions in such a way that $\mathcal{A}_{1-3}$ are still not violated by the protocol. The rigorous discussion of the steps we have taken would require the introduction of several new auxiliary variables and other formal details ${ }^{\dagger}$ what we do not do here. What comes below is just the brief outline of the strategy we used.

The assertions $\mathcal{A}_{4-11}$ generated during the verification of unbounded SCMP were used as a guideline to determine the weaker requirements. We kept all the CI requirements generated by (1) except the ones for the R.RecvPkt event.

We can see that $\mathcal{A}_{4-6}$ are not affected by using modulo- $N$ timestamps. The crucial assertion affected by the use of modulo- $N$ timestamps is $\mathcal{A}_{7}$ which claims that the history variable $t s_{-} r c v d$ of the server contains monotone increasing timestamps. Thus we have to assure that this assertion is preserved by R.RecvPkt.

From the specification of the R.ClkTick event, we can derive bounds on the range of

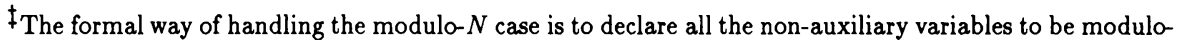
$N$, introduce an unbounded auxiliary variable for each and prove that they are always equal modulo- $N$. To define the real-time properties of the system, we also have to introduce various epoch variables and formulate real-time assumptions (see e.g. Shankar, 1994).
} 
the modulo- $N$ timestamps that may be acceptable to the server. This range is given by $\left[S . t i m e-W_{S}, S . t i m e+\epsilon\right]$. What we have to prove is that any request in the network which has an older timestamp than the timestamp of the last accepted request, must have a timestamp that falls outside of the modulo- $N$ interval given above. To prove this, we can use the following facts:

- If $t$ and $u$ are the timestamps of the most recent and the oldest request in the network, respectively, then $t-u$ is bounded because of the bounded lifetime of client incarnations and packets in the network.

- When the most recent request was accepted by the server, its timestamp must have fallen into the $\left[\right.$ S.time $-W_{S}$, S.time $\left.+\epsilon\right]$ interval.

- Because the rate of the server clock is bounded, requests older than the most recent accepted will disappear from the network before the $\left[\right.$ S.time $\left.-W_{S}, S . t i m e+\epsilon\right]$ interval wraps around.

Based on this strategy, we have derived that R.RecvPkt preserves $\mathcal{A}_{7}$ provided that

$N>\left\lceil\frac{M P L}{\gamma}\right\rceil+W_{C}+W_{S}+\epsilon+1$

where $M P L$ is the maximum packet lifetime, $\gamma$ is the minimum time between two clock ticks (thus $1 / \gamma$ is the maximum clock rate). $W_{C}, W_{S}, \epsilon$ are parameters from the protocol specification, namely maximum lifetime of a client incarnation, server incarnation and estimated difference between clocks, respectively, all measured in clock ticks.

The CI condition for the C.RecvPkt event using (1) is

$\square(A \in z \Rightarrow C . C R(A . d s t, A . s r c) . t s \geq A . t s \geq C . C R(A . d s t, A . s r c) . t s-N+1)$

Notice that we use $K=0$ here because it is easy to show that $C . C R(A . d s t, A . s r c) . t s \geq A$.ts always holds. It can be proven that this $\mathrm{CI}$ condition is satisfied if the following inequality holds:

$N>W_{C}+\left\lceil\frac{2 M P L+W_{S} \cdot \Gamma}{\gamma}\right\rceil$

where $\Gamma$ is the maximum time between clock ticks.

To summarize our results, we have proven that SCMP satisfies the safety assertions $\mathcal{A}_{1-3}$ when timestamps and clocks are modulo- $N$ identifiers provided that $N$ satisfies the inequalities (2) and (3). These inequalities determine the minimal safe value of $N$ from system parameters such as the bounds on the clock rates and the maximum packet lifetime.

\section{MODELING OF FAILURES}

A CM protocol must be prepared for the failure of hosts which is usually called a crash. When a host crashes, it loses the contents of its memory and stops working. The actions 
Crash $\{$ The client and server events are identical. $\}$

ec: $\quad$ rstatus $=$ running

ac: $\quad$ rstatus $:=$ down;

C.Recover

ec: $\quad$ rstatus $=$ down and time has advanced since the crash

ac: $\quad$ rstatus := running;

for each $C R(a, b)$

$C R(a, b)$. status $:=$ closed;

\section{R.Recover}

ec: $\quad$ rstatus $=$ down

ac: $\quad$ rstatus $:=$ running;

upper $:=$ time $+\epsilon$

for each $C R(a, b)$

$C R(a, b)$. status $:=$ closed;

Table 3 Crash recovery at the client and at the server

taken by a host to recover from crashes should preserve the safety requirements and should assure that normal communication commences as soon as possible.

To model a crash, we have to introduce a new state variable rstatus which can be either running or down; and two new events Crash and Recover. Then we add rstatus = running to the enabling condition of the events in Table 1 and 2 except the ClkTick events. We assume that the clocks do not stop working, or if they do then they can be reinitialized after the crash.

The specification of the Crash and Recover events is in Table 3. Crash simply sets rstatus to down for both clients and servers. A client is only allowed to recover from a crash when its clock advanced at least by one tick. This restriction is necessary because the status of every connection is set to closed during the recovery and assertion $\mathcal{A}_{9}$ has to be preserved.

A server is allowed to recover immediately after a crash. Setting upper to time $+\epsilon$, the monotonicity of time and the assertion below

$\square\left(S . C R(a, b) . t s \_r c v d(S . C R(a, b)\right.$. lin $) \leq$ time $\left.+\epsilon\right)$

assure that $\mathcal{A}_{11}$ will be preserved by the recovery procedure thus no duplicate requests will be accepted.

Based on the above hints, it is trivial to extend the verifications of Section 3 and 4 for the protocol which models crashes. The requirements for $N$, Equations (2) and (3) in Section 4, remain valid. There is another crash-recovery mechanism discussed by (Liskov et al, 1991) which requires safe storage for a server state variable. That mechanism can be modeled in a similar way. 


\section{CONCLUSION}

We have presented the verification of a connection management protocol (Liskov et al, 1991). The protocol uses monotonic clocks and timestamps to provide reliable connection: management over an unreliable bounded-delay network. We have proven that the safety of the protocol does not require synchronized clocks even in the case when modulo- $N$ timestamps are used although valid connection requests may be rejected when the clocks are not synchronized. Expressions for the minimum safe value of $N$ have been derived from the parameters of the network.

Assertional reasoning based on temporal logic (Shankar, 1993) has been used to verify the protocol. We combined standard techniques from the literature (Lamport, 1994), (Shankar, 1993) with ad hoc methods to generate the proofs. In our experience, the verification process requires lots of intuition and a thorough understanding of the analyzed protocol. We plan to document our proofs in a machine-readable format in order to be able to validate them with an automatic proof-checker.

\section{REFERENCES}

Biersack, E. W and Feldmeier, D. C. (1993) A timer-based connection management protocol with synchronized clocks and its verification. Computer Networks and ISDN Systems, 25(12), 1303-1319.

Fletcher, J. G. and Watson, R. W. (1978) Mechanisms for a reliable timer-based protocol. Computer Networks, 2, 271-290.

Lamport, L. (1994) Verification and specification of concurrent programs, in J. W. de Bakker, W.-P. de Roever, and G. Rozenberg, editors, A Decade of Concurrency: Reflections and Perspectives, volume 803 of Lecture Notes in Computer Science, pages 347-374.

Lampson, B. W., Lynch, N. A. and Sogaard-Andersen, J. F. (1993) Correctness of atmost-once message delivery protocols, in FORTE'93, pages 387-402.

Liskov, B., Shrira, L. and Wroclawski, J. (1991) Efficient at-most-once messages based on synchronized clocks. ACM Transactions on Computer Systems, 9(2), 125-142.

Oláh, A. L. and Heemstra de Groot, S. M. (1995) Assertional verification of a connection management protocol. Memoranda Informatica 95-28, University of Twente.

Shankar, A. U. (1991) Modular design principles for protocols with an application to the transport layer. Proceedings of the IEEE, 79(12), 1687-1707.

Shankar, A. U. (1993) An introduction to assertional reasoning for concurrent systems. ACM Computing Surveys, 25(3), 225-262.

Shankar, A. U. (1993) Reasoning assertionally about real-time systems. Proceedings of the IEEE, 82(1), 172-183.

Shankar, A. U. and Lee, D. (1993) Modulo- $N$ incarnation numbers for cache-based transport protocols. In International Conference on Network Protocols, pages 46-54, San Francisco, CA, USA.

Sunshine, C. A. and Dalal, Y. K. (1978) Connection management in transport protocols. Computer Networks, 2, 454-473.

Watson, R. W. (1981) Timer-based mechanisms in reliable transport protocol connection management. Computer Networks, 5, 47-56. 


\section{BIOGRAPHY}

András Oláh studied electrical engineering at the Technical University of Budapest, Hungary. He received the M.Sc. degree in 1991. Since 1992, he has been a Ph.D. student in the group of Tele-Informatics and Open Systems at the University of Twente, the Netherlands. His research interests are the design and implementation of computer communication protocols.

Sonia Heemstra de Groot studied electrical engineering at Mar del Plata National University, from which she graduated in 1983. She received a second master degree in electrical engineering from the NUFFIC in 1986 and a Ph.D. in electrical engineering from the University of Twente, The Netherlands, in 1990. From 1986 to 1990 she worked as an assistant researcher in the group for Network Theory and VLSI Design of the Department of Electrical Engineering of the University of Twente, concentrating her attention on topics related to real-time processing of DSP algorithms and efficient multiprocessor scheduling. In 1991 she became a lecturer at the group Tele-Informatics and Open Systems of the same department. Her research interests include different aspects of protocol design and implementation, in particular time-critical protocol functions and resource allocation and control. 